\title{
Correlation Between Genetic factors and the Incidence of ocular Hypertension Glaucoma / Normal tension Glaucoma
}

\author{
Yali Feng \\ Department of Ophthalmology \\ the Third Affiliated Hospital of Qiqihar Medical \\ University \\ Qiqihar, China \\ fengyl151129@126.com \\ Wenshuang $\mathrm{Xu}$ \\ Department of Ophthalmology \\ I Qiqihar Otorhinolaryngology Hospital \\ Qiqihar, China \\ zxf2015getup@126.com
}

\author{
Zhe Cui \\ Department of Ophthalmology \\ the Third Affiliated Hospital of Qiqihar Medical \\ University \\ Qiqihar, China \\ everyone2014good@qq.com
}

Keywords: Genetic factors; high tension glaucoma; normal tension glaucoma; SNP; polymorphism .

\begin{abstract}
Objective To explore the correlation between genetic factors and incidence of primary open angle glaucoma (POAG) Method 1157 cases of patients with POAG composed by 297 NTG and 860 HTG were recruited. Genomic DNA from each case was genotyped for 13 loci of SNPs in 4 genes ( TMCO1, CDKN2B-AS1, ATOH7 and SIX1/SIX6. Relationship between SNP allelic frequency and the clinical indexes was calculated. Results The variation of rs4656461 and rs7555523 in TMCO1 were susceptible to the onset of POAG, while the variation of rs1063192, rs523096, rs7049105, rs2157719 in CDKN2B-AS1, rs33912345, rs10483727 in SIX1 / SIX6 related to POAG as protective factors. Variation of rs4656461 and rs7555523 in TMCO1 was susceptible to HTG, while rs523096, rs2157719 in CDKN2B-AS1 and rs33912345, rs10483727 in SIX1 / SIX6 correlated with NTG more protectively, IOP correlated with SNPs of rs523096, rs2157719 in CDKN2B-AS1 and rs33912345, rs10483727 in SIX1 / SIX6 with either multiple correction of age and gender, or Bonferroni adjustment. Conclusion The polymorphism of rs523096, rs2157719 in CDKN2B-AS1 and rs33912345, rs10483727 in SIX1 / SIX6 correlated with NTG significantly, and polymorphism of rs4656461 and rs7555523 in TMCO1 was susceptible to HTG.
\end{abstract}

\section{Introduction}

Primary open angle glaucoma (POAG) is the most common type of glaucoma in China, and is one of the main causes of blindness related to optic nerve injury.[1] In clinic, POAG is composed of high tension glaucoma(HTG) and normal tension glaucoma(NTG), due to the high risk factor of intraocular hypertension, belonging to polygenic diseases with obvious tendency of heredity. As Genome wide association studies (GWAS) development, the genetic mechanism of complicated polygenic diseases such as POAG is hopefully to be learned [2]. There are many researches have demonstrated the polymorphism of several genes related to common complex phenotypes of POAG, such as CAV1/CAV2, ATOH7, TMCO1, CDKN2B-AS1, SIX1/SIX6. Burdon et al[3]. has demonstrated the variation of the susceptibility genes of TMCO1 and CDKN2B-AS1 related to glaucoma, Wiggs et al[4] pointed CDKN2B-AS1 and SIX1/SIX6 susceptible POGA to NTG. Therefore, the purpose of this article is to verify the relationship between SNPs in above-mentioned genes and POAG patients in China, and explore the correlation of different genotypes with deferent phenotype of POAG. 


\section{Materials and Methods}

Clinical Subjects. This study was approved by the ethics committee of our hospital, and all procedures are in line with the regulations, and informed consent was obtained from all subjects. 1157 cases of POAG were enrolled. All subjects received the complete examination of the best corrected visual acuity, slit lamp anterior chamber examination, intraocular pressure measurement and fundus examination. Besides, the central corneal thickness (CCT), ocular axial length (AL), anterior chamber angle, visual field (VF) was examined in the patients with suspected glaucoma. According to intra-ocular pressure, the POAG patients were divided into two subgroups as HTG (maximum intraocular pressure $>21 \mathrm{mmHg}$ ) and NTG (maximum intraocular pressure $\leq 21 \mathrm{mmHg}$ ), composed by 297 and 860 cases respectively. 934 cases of healthy volunteers were recruited as control group, who met the grouping criteria of binocular intraocular pressure lower than $21 \mathrm{mmHg}$, and without glaucomatous optic neuropathy and immediate family history of glaucoma.

Inclusion and Exclusion Criteria. The patient needs to meet the following criteria as glaucomatous optic neuropathy for at least one eye or optic nerve injury visual defect for at least one eye. Glaucomatous optic neuropathy was defined that nerve fiber layer defects, glaucoma visual field defects or gonioscopy for open angle lead by vertical cup to disc ratio (VCDR) was larger than 0.7 or focal lesion.

Exclusion criteria were with any history of secondary glaucoma, ocular trauma, extensive use of glucocorticoid in the whole or part of body.

Genetic Typing. $5 \mathrm{ml}$ peripheral blood from each subject was drawn to extract genome DNA. The DNA was purified by Qiagen QIAmp kit. iPLEX Gold chemistry Mass ARRAY system (Sequenom, SanDiego, California) was used for SNPs genotyping, checked by Matrix assisted laser desorption ionization time of flight mass spectrometry (MALDI-TOF) .The SNPs included the loci that rs4656461, rs7555523 in TMCO1, rs1063192, rs523096, rs7049105, rs2157719, rs4977756, rs10116277 in CDKN2B-AS1, rs7916697, rs1900004, rs3858145 in ATOH7, and rs33912345, rs10483727 in SIX1/SIX6, which reviewed from articles.

Statistical Analysis. Continuous variables are expressed as mean \pm SD. Software SPSS v.13.0 was used for data statistical analysis. PLINK1.07 was applied for genotypic analysis. Odds ratio (OR) and $95 \%$ confidence interval (CI) were calculated by Logistic regression, Linear regression with additional genetic model was analyzed for correlation between SNP polymorphism and the indexes of age, CCT, intraocular pressure, AL and VCDR. The comparison between groups was calculated by $\mathrm{t}$ test, and Bonferroni adjustment was uses for correction for multiple comparisons.

\section{Results}

General Information. The general information of 1157 cases and 934 controls was listed in Table 1 , and information of POAG subgroups was listed in Table 2. The factors of age and gender were adjusted in the subsequent analysis. 
TABLE I. GENERAL INFORMATION OF POAG GROUP AND CONTROL GROUP

\begin{tabular}{|c|c|c|}
\hline & POAG Group & Control Group \\
\hline Cases & 1157 & 934 \\
\hline Female $\%$ & $32.24 \% *$ & $59.74 \%$ \\
\hline Age & $48.81 \pm 16.28 *$ & $53.37 \pm 14.83$ \\
\hline Max IOP(mmHg) & $30.10 \pm 11.37 *$ & $14.85 \pm 2.84$ \\
\hline VCDR & $0.85 \pm 0.12 *$ & $0.34 \pm 0.11$ \\
\hline
\end{tabular}

TABLE II. CLINICAL SYMPTOMS OF HTG AND NTG SUBGROUPS

\begin{tabular}{|c|c|c|}
\hline & HTG & NTG \\
\hline Cases & 860 & 297 \\
\hline Female $\%$ & $29.77 \% *$ & $39.53 \%$ \\
\hline Age & $42.14 \pm 16.49 *$ & $49.92 \pm 14.41$ \\
\hline Max IOP $(\mathrm{mmHg})$ & $33.75 \pm 10.60 *$ & $18.47 \pm 2.22$ \\
\hline CCT & $545.04 \pm 35.52 *$ & $528.37 \pm 34.99$ \\
\hline AL & $24.93 \pm 1.70$ & $24.87 \pm 1.62$ \\
\hline Max VCDR & $0.85 \pm 0.12$ & $0.83 \pm 0.11$ \\
\hline \multicolumn{2}{|l|}{} \\
* indicated difference with statistical significance $(\mathrm{p}<0.05)$
\end{tabular}

Correlation of Allele frequency and POAG . The quality control and genotypic efficiency of SNP polymorphism could reach more than 95\%. There were no statistical difference between two groups by Hardy-Weinberg Examination (P>0.05). The genotypes of 13 SNP loci were listed in Table3. By adjustment of age and gender, the variation of rs4656461 and rs7555523 in TMCO1 were susceptible to the onset of POAG, while the variation of rs1063192, rs523096, rs7049105, rs2157719 in CDKN2B-AS1, rs33912345, rs10483727 in SIX1 / SIX6 related to POAG as protective factors. After Bonferroni adjustment, rs4656461and rs7555523 of TMCO1, rs2157719and rs523096 of CDKN2B-AS1, rs33912345 and rs10483727 of SIX1/SIX6 still showed a significant correlation with POAG

Correlation of Allele Frequency in Subgroups. It was listed in Table 4 that allele frequency distribution in HTG and NTG subgroups. Variation of rs4656461 and rs7555523 in TMCO1 was susceptible to HTG, and variation of rs523096, rs7049105, rs2157719 in CDKN2B-AS1 and rs33912345, rs10483727 in SIX1 / SIX6 related to HTG as protective factors. After Bonferroni adjustment, rs4656461, rs7555523 in TMCO1 and rs523096, rs2157719 in CDKN2B-AS1 still showed a significant correlation with HTG.

$P$ value was less in NTG subgroup compared with NTG subgroup and control group with rs523096, rs2157719 in CDKN2B-AS1 and rs33912345, rs1048372. 
TABLE III. CORRELATION OF ALLELE FREQUENCY OF 13 SNPS LOCI WITH POAG

\begin{tabular}{|c|c|c|c|c|c|c|c|c|}
\hline Gene & CHR & SNP & $\mathbf{B P}$ & MA & MAF_case & MAF_Control & OR & $\mathbf{P}$ \\
\hline TMCO1 & 1 & rs4656461 & 165687205 & G & 0.018 & 0.005 & $3.18(1.52-6.65)$ & 0.002 \\
\hline TMCO1 & 1 & rs 7555523 & 165718979 & $\mathrm{C}$ & 0.016 & 0.005 & $3.31(1.52-7.20)$ & 0.003 \\
\hline CDKN2B-AS1 & 9 & rs 1063192 & 22003367 & $\mathrm{C}$ & 0.184 & 0.204 & $0.85(0.72-1.00)$ & 0.047 \\
\hline CDKN2B-AS1 & 9 & rs523096 & 22019129 & $\mathrm{C}$ & 0.095 & 0.135 & $0.65(0.53-0.80)$ & $5.833 \times 10^{-5}$ \\
\hline CDKN2B-AS1 & 9 & rs7049105 & 22028801 & A & 0.319 & 0.361 & $0.82(0.71-0.94)$ & 0.006 \\
\hline CDKN2B-AS1 & 9 & rs2157719 & 22033366 & G & 0.092 & 0.133 & $0.64(0.52-0.79)$ & $3.528 \times 10^{-5}$ \\
\hline CDKN2B-AS1 & 9 & rs 4977756 & 22068652 & G & 0.214 & 0.227 & $0.89(0.76-1.04)$ & 0.128 \\
\hline CDKN2B-AS1 & 9 & rs1011627 & 722081397 & G & 0.302 & 0.311 & $0.95(0.82-1.09)$ & 0.454 \\
\hline ATOH7 & 10 & rs7916697 & 69991853 & A & 0.388 & 0.391 & $0.97(0.85-1.11)$ & 0.661 \\
\hline ATOH7 & 10 & rs 1900004 & 70000881 & A & 0.389 & 0.391 & $0.97(0.84-1.11)$ & 0.651 \\
\hline ATOH7 & 10 & rs3858145 & 70011838 & G & 0.385 & 0.393 & $0.95(0.83-1.09)$ & 0.445 \\
\hline SIX1/SIX6 & 14 & rs33912345 & 60976537 & A & 0.201 & 0.251 & $0.75(0.64-0.88)$ & $5.503 \times 10^{-4}$ \\
\hline SIX1/SIX6 & 14 & rs 10483727 & 61072875 & $\mathrm{C}$ & 0.209 & 0.254 & $0.78(0.67-0.92)$ & 0.003 \\
\hline
\end{tabular}

\begin{tabular}{|c|c|c|c|c|c|c|c|c|c|}
\hline \multirow[b]{2}{*}{ Gene } & \multirow[b]{2}{*}{ SNP } & \multirow[b]{2}{*}{ MA } & \multirow{2}{*}{$\begin{array}{c}\text { MAF } \\
\text { Control }\end{array}$} & \multicolumn{3}{|c|}{ HTG } & \multicolumn{3}{|c|}{ NTG } \\
\hline & & & & $\begin{array}{l}\text { MA } \\
\text { case }\end{array}$ & $O R(95 \% C I)$ & $P$ & $\begin{array}{l}\text { MA } \\
\text { case }\end{array}$ & $O R(95 \% C I)$ & $P$ \\
\hline TMCO1 & rs4656461 & $\mathrm{G}$ & 0.005 & 0.02 & $3.70(1.72-7.97)$ & $8.465 \times 10^{-4}$ & 0.014 & $2.55(0.96-6.80)$ & 0.062 \\
\hline TMCO1 & rs 7555523 & $\mathrm{C}$ & 0.005 & 0.018 & $3.85(1.72-8.62)$ & 0.001 & 0.012 & $2.61(0.92-7.35)$ & 0.071 \\
\hline CDKN2B-AS1 & rs1063192 & $\mathrm{C}$ & 0.204 & 0.186 & $0.88(0.73-1.05)$ & 0.146 & 0.177 & $0.79(0.61-1.01)$ & 0.063 \\
\hline CDKN2B-AS1 & rs523096 & $\mathrm{C}$ & 0.135 & 0.101 & $0.70(0.56-0.87)$ & 0.002 & 0.078 & $0.52(0.36-0.73)$ & $1.765 \times 10^{-4}$ \\
\hline CDKN2B-AS1 & rs7049105 & $\mathrm{A}$ & 0.361 & 0.32 & $0.84(0.72-0.98)$ & 0.029 & 0.314 & $0.79(0.64-0.98)$ & 0.033 \\
\hline CDKN2B-AS1 & rs2157719 & $\mathrm{G}$ & 0.133 & 0.099 & $0.70(0.56-0.88)$ & 0.002 & 0.071 & $0.4(0.33-0.67)$ & $3.445 \times 10^{-5}$ \\
\hline CDKN2B-AS1 & rs4977756 & $\mathrm{G}$ & 0.227 & 0.213 & $0.90(0.76-1.07)$ & 0.235 & 0.218 & $0.88(0.69-1.11)$ & 0.28 \\
\hline CDKN2B-AS1 & rs10116277 & $\mathrm{G}$ & 0.311 & 0.306 & $0.98(0.84-1.15)$ & 0.818 & 0.289 & $0.87(0.70-1.08)$ & 0.2 \\
\hline ATOH7 & rs7916697 & $\mathrm{A}$ & 0.391 & 0.398 & $1.02(0.88-1.18)$ & 0.797 & 0.356 & $0.83(0.68-1.02)$ & 0.075 \\
\hline ATOH7 & rs1900004 & A & 0.391 & 0.399 & $1.02(0.88-1.19)$ & 0.784 & 0.356 & $0.83(0.68-1.02)$ & 0.073 \\
\hline ATOH7 & rs3858145 & $\mathrm{G}$ & 0.393 & 0.397 & $1.00(0.86-1.16)$ & 0.998 & 0.35 & $0.79(0.65-0.98)$ & 0.029 \\
\hline SIX1/SIX6 & rs33912345 & $\mathrm{A}$ & 0.251 & 0.21 & $0.80(0.68-0.96)$ & 0.015 & 0.176 & $0.64(0.50-0.82)$ & $4.288 \times 10^{-4}$ \\
\hline
\end{tabular}

TABLE IV. LINEAR REGRESSION ANALYSIS OF MAXIMAL IOP AND SNPS

\begin{tabular}{|c|c|c|c|c|}
\hline \multirow{2}{*}{ Gene } & \multirow{2}{*}{ SNP } & \multirow{2}{*}{ MA } & \multicolumn{2}{|c|}{ IOP } \\
\hline & & & BETA & $P$ \\
\hline TMCO1 & rs4656461 & G & -0.153 & 0.927 \\
\hline TMCO1 & rs7555523 & $\mathrm{C}$ & 0.622 & 0.723 \\
\hline CDKN2B-AS1 & rs1063192 & $\mathrm{C}$ & 1.387 & 0.016 \\
\hline CDKN2B-AS1 & rs523096 & $\mathrm{C}$ & 2.4 & 0.001 \\
\hline CDKN2B-AS1 & rs7049105 & $\mathrm{A}$ & 0.538 & 0.265 \\
\hline CDKN2B-AS1 & rs2157719 & G & 2.367 & 0.002 \\
\hline CDKN2B-AS1 & rs4977756 & $\mathrm{G}$ & 1.118 & 0.041 \\
\hline CDKN2B-AS1 & rs10116277 & G & 0.965 & 0.056 \\
\hline ATOH7 & rs7916697 & A & 0.391 & 0.407 \\
\hline ATOH7 & rs1900004 & A & 0.333 & 0.481 \\
\hline ATOH7 & rs3858145 & G & 0.318 & 0.501 \\
\hline SIX1/SIX6 & rs33912345 & A & 1.748 & 0.002 \\
\hline SIX1/SIX6 & rs10483727 & $\mathrm{C}$ & 1.742 & 0.002 \\
\hline
\end{tabular}


in SIX1 / SIX6, indicated these loci correlated with NTG more protectively. All 4 SNPs were still with significance after Bonferroni adjustment. .

Correlation of SNP polymorphism with Clinical Symptoms. The correlation was analyzed between 13 SNP polymorphism and 4 clinical symptoms of IOP, CCT, AL, and VCDR. After adjustment of age and gender, only IOP correlated with SNPs of rs523096, rs2157719 in CDKN2BAS1 and rs33912345, rs10483727 in SIX1 / SIX6 (Table 5). Results by Bonferroni adjustment also showed correlation for these 4 SNPs.

\section{Discussions}

Glaucoma is a serious disease of blindness, it is estimated that the patients with which will be reached 80 million by 2020[5]. Glaucoma is a kind of genetic heterogeneity disease, causing neurodegenerative disease, resulting vision loss and other serious consequences. The characters of neurodegenerative disease often manifest as degeneration of the optic nerve, the change of visual field and the loss of retinal ganglion cells [6]. According to pathogeny, Glaucoma is composed of primary and secondary type, and according to phenotype, it is composed of HTG and NTG. Current clinical data suggest that the increase of intraocular pressure is the main risk factor for glaucoma. So far, at least 22 genes and numerous SNP loci were found to correlate with POAG. Genetic factors play an important role in the pathogenesis of POAG [7].

In this study, multiple SNPs in genes of TMCO1, CDKN2B-AS1, ATOH7 and SIX1/SIX6 were analyzed with POAG. The loci of rs4656461, rs7555523 in TMCO1, and rs33912345, rs10483727 in SIX1/SIX6 showed statistical correlation with POAG, either with multiple correction of age and gender, or Bonferroni adjustment. Pasquale et al found CDKN2B-AS1 related to NTG in American people, and the variation was not found in HTG. But in our study, the SNPs in that gene related both types of POAG, only related to NTG. It may resulted by the distribution of clinical samples and race region.

SIX1/SIX6 is the gene earliest reported related to POAG, which increases VCDR significantly and the risk of onset of POAG. In this study, rs10483727and rs33912345, located in SIX1/SIX6, exerts relatively strong correlation, especially in NTG. It suggests SIX1/SIX6 may impact the clinical phenotype of patients, can be used in early diagnosis and treatment, and clinical medication guidance in NTG.

\section{Conclusions}

In Han group, TMCO1, CDKN2B-AS1 and SIX1/SIX6 related to POAG. The polymorphism rs523096, rs2157719 in CDKN2B-AS1 and rs33912345, rs10483727 in SIX1 / SIX6 correlated with NTG significantly, and polymorphism of rs4656461 and rs7555523 in TMCO1 was susceptible to HTG. Therefore, the polymorphism of these loci can be used in clinic to predict the risk and type of POAG in early diagnosis and treatment, and clinical medication guidance.

\section{Acknowledgment}

This study is support by the program of the Education Department of Heilongjiang Province , China. The number of project is 12521625 .

\section{References}

[1] Tham, Y.C., et al., Aggregate Effects of Intraocular Pressure and Cup-to-Disc Ratio Genetic Variants on Glaucoma in a Multiethnic Asian Population. Ophthalmology, 2015, vol 122 ,pp:1149-1157

[2] Springelkamp, H., et al., ARHGEF12 influences the risk of glaucoma by increasing intraocular pressure. Hum Mol Genet, 2015, 24(9):.1-11 
[3] Burdon KP, Macgregor S, Hewitt AW, et al.. Genome-wide association study identifies susceptibility loci for open angle glaucoma at TMCO1 and CDKN2B-AS1. Nat Genet. 2011; 43(6):574-578.

[4] Wiggs JL, Yaspan BL, Hauser MA, et al. Common variants at 9p21 and 8q22 are associated with increasedsusceptibility to optic nerve degeneration in glaucoma. PLoS Genet. 2012b; 8(4):e1002654

[5] Pasquale, L.R., et al., CDKN2B-AS1 genotype-glaucoma feature correlations in primary openangle glaucoma patients from the United States. Am J Ophthalmol, 2013. 155(2): p. 342-353 e5.

[6] Abu-Amero, K.K., et al., Lack of association of SNP rs4236601 near CAV1 and CAV2 with POAG in a Saudi cohort. Mol Vis, 2012. 18: p. 1960-5..

[7] Park, S., et al., Common TGFbeta2, BMP4, and FOXC1 variants are not associated with primary open-angle glaucoma. Mol Vis, 2012. 18: p. 1526-39.

[8] Mabuchi, F., et al., Association between genetic variants associated with vertical cup-to-disc ratio and phenotypic features of primary open-angle glaucoma. Ophthalmology, 2012. 119(9): p. 1819-25.

[9] Sugiyama, K., [A challenge to primary open-angle glaucoma including normal-pressure. Clinical problems and their scientific solution]. Nihon GankaGakkaiZasshi, 2012. 116(3): p. 233-67; discussion 268.

[10] Williams, S.E., et al., The genetics of POAG in black South Africans: a candidate gene association study. Sci Rep, 2015. 5: p. 8378. 